\title{
Adjustments and Uncertainty Quantification for SLS Aerodynamic Sectional Loads
}

Derek J. Dalle, Stuart E. Rogers, NASA Ames Research Center

Henry C. Lee, Jamie G. Meeroff

Science \& Technology Corp.

Computational Aerosciences Branch

NASA Advanced Supercomputing Division (ARC/TNA)

2018 AIAA Aviation Forum

June 27, 2018 


\section{The Space Launch System (SLS): EM-1 mission map}

\section{EXPLORATION MISSION-1}

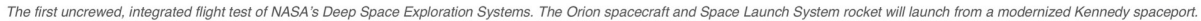

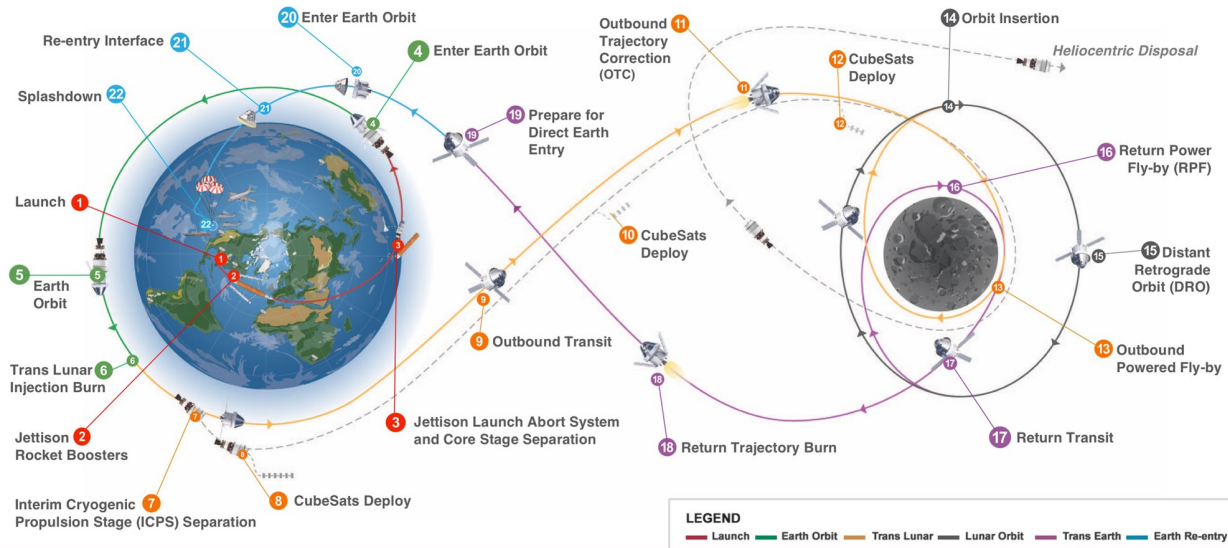

Total distance traveled: 1.3 million miles - Mission duration: 25.5 days - Re-entry speed: 24,500 mph (Mach 32) - 13 CubeSats deployed 


\section{Space Launch System multiple configurations}

$322 \mathrm{ft}$

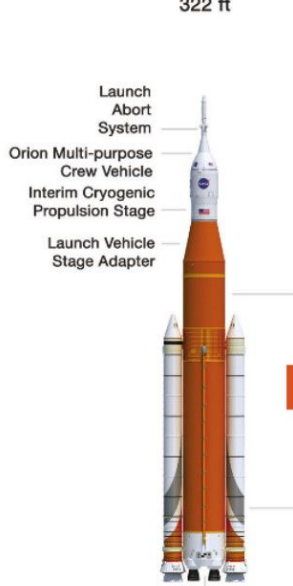

SLS Block 1
$364 \mathrm{ft}$

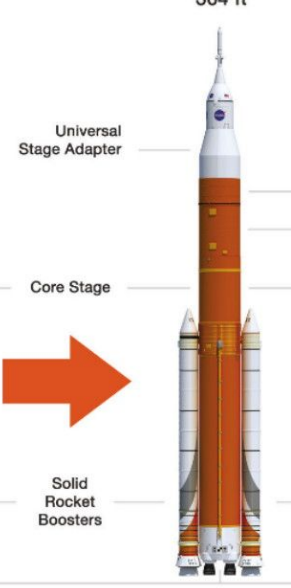

SLS Block 1B Crew
$327 \mathrm{ft}$

$365 \mathrm{ft}$

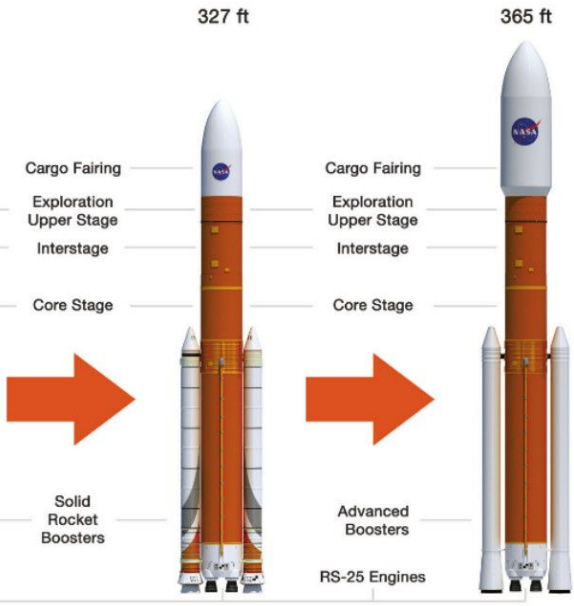

SLS Block 1B Cargo

SLS Block 2 Cargo 


\section{Sectional Loads/Line Loads}

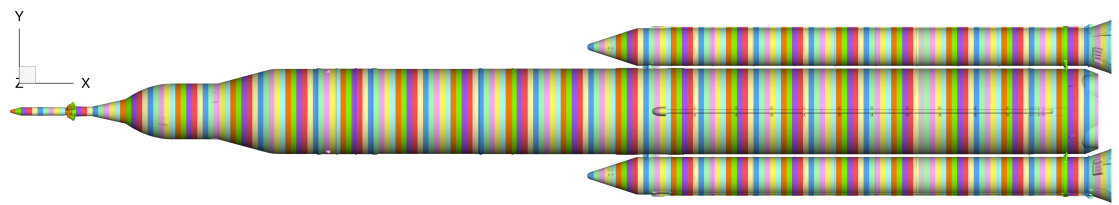

Sectional load slices for SLS Block 1B Crew configuration
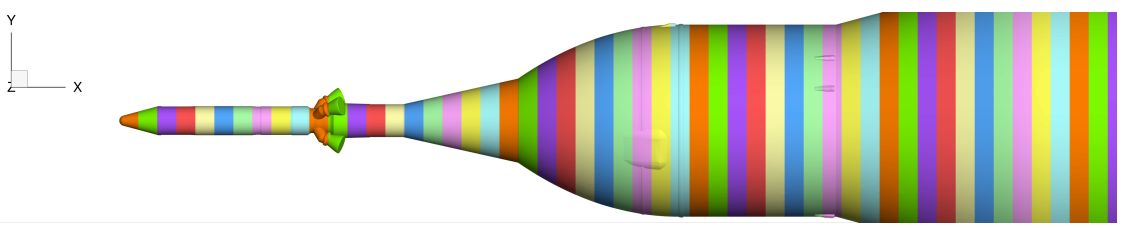

Sectional load slices on forward portion of SLS Block 1B

- Line loads are a simple tool to interface aero loads and vehicle structures by dividing vehicle into a number of slices

- Calculate the load on each slice

- Valid for long/skinny vehicles 


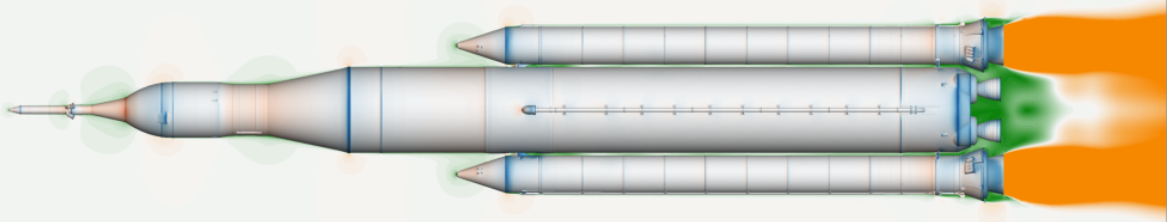

Mach 0.50 


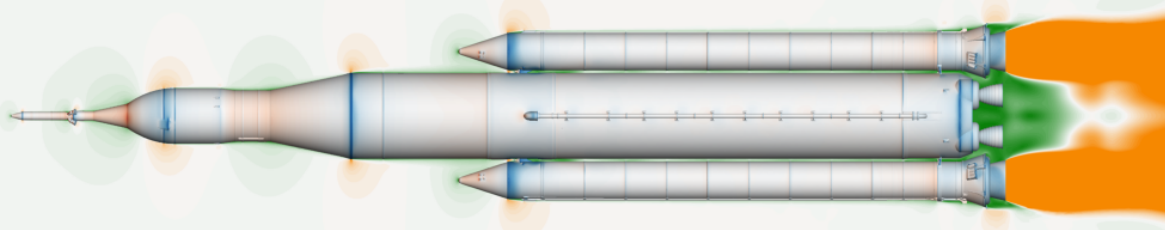

Mach 0.70 




Mach 0.80 


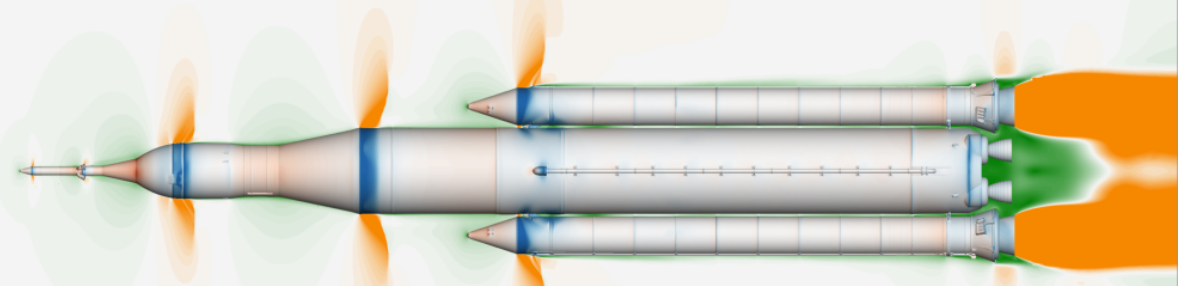

Mach 0.90 
Mach 0.95 
Mach 0.98 
Mach 1.00 
Mach 1.05 
Mach 1.10 
Mach 1.20 
Mach 1.30 





Mach 1.60 
Mach 1.75 
Mach 2.00 
Mach 2.50 
Mach 3.00 
Mach 3.50 
Mach 4.00 
Mach 1.75 


\section{What Does a Sectional Load Look Like?}



The load is basically a set of 3 discretized curves, $c_{A}(x), c_{Y}(x)$, and $c_{N}(x)$ This example is from SLS Block 1B, Mach 1.75, $\alpha=4^{\circ}, \beta=0^{\circ}$ 


\section{Example of a Sectional Load}

- Three force components each have a profile as a function of axial distance along the rocket

- The dimensional version of this are force per length, e.g. Ibf/in

- For SLS, we use 200 slices and deliver line loads on the core, left booster, and right booster all separately

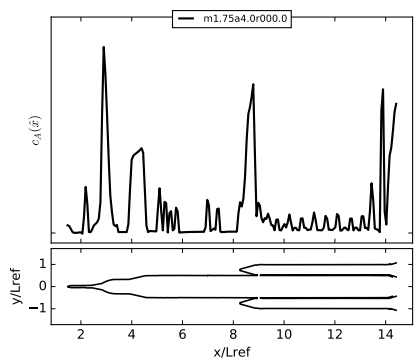

Axial loads: $c_{A}\left(x / L_{r e f}\right)=c_{A}(\hat{x})$



Lateral loads: $c_{Y}(\hat{x})$

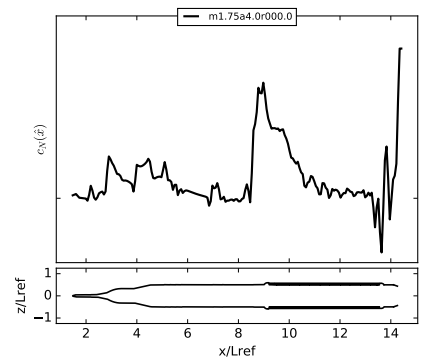

Normal loads: $c_{N}(\hat{x})$ 


\section{Sample Sectional Normal Loads on SLS Block 1B}

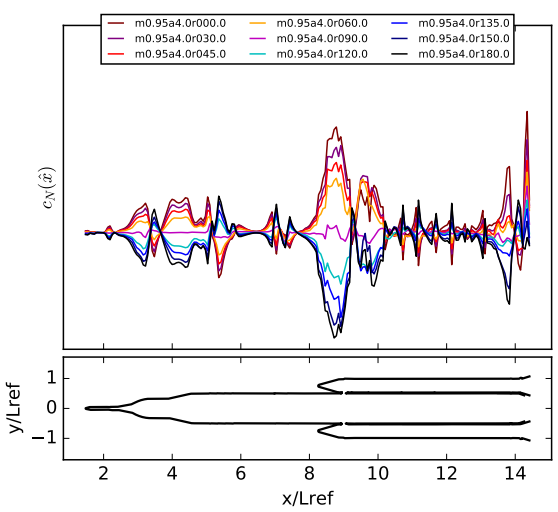

Mach 0.95, $\alpha_{t}=4^{\circ}, \beta \geq 0^{\circ}$
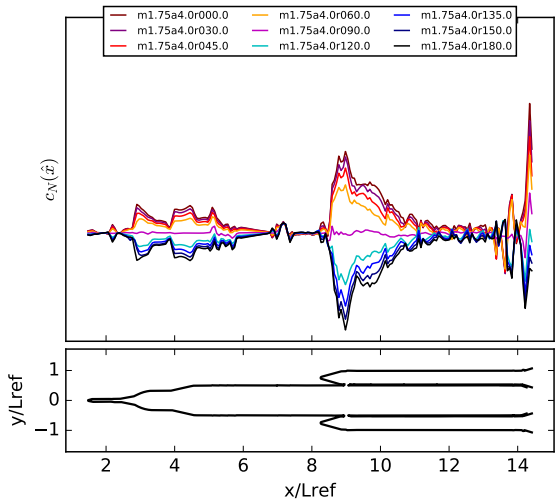

Mach 1.75, $\alpha_{t}=4^{\circ}, \beta \geq 0^{\circ}$

- Each plot contains 9 sectional loads at the maximum angle between the nose and the velocity

- The load profiles change quite a bit with Mach number

- At one Mach number, load is just about proportional to $\alpha$ 


\section{Motivation for Adjustments}

\section{Inconsistency}

The line load profiles should have certain integral properties:

$$
C_{N}=\int_{\hat{x}_{1}}^{\hat{x}_{2}} c_{N}(\hat{x}) \mathrm{d} \hat{x} \quad C_{m}=\int_{\hat{x}_{1}}^{\hat{x}_{2}}\left(\hat{x}-\hat{x}_{M R P}\right) c_{N}(\hat{x}) \mathrm{d} \hat{x}
$$

However, frequently the line loads $c_{N}(\hat{x})$ come from CFD because of the higher density of data, while the force $\&$ moment database is derived from wind tunnel testing

How can we adjust the profile to create $\bar{c}_{N}(\hat{x})$ that's consistent the integral constraints above?

\section{Uncertainty Quantification}

It is easy (and common practice) to disperse the integrated forces and moments:

$$
\tilde{C}_{N}=C_{N}+\varepsilon_{C N} U_{C N} \quad \tilde{C}_{m}=C_{m}+\varepsilon_{C L M} U_{C L M}+\left(\hat{x}_{M R P}-\hat{x}_{C g}\right) \varepsilon_{C N} U_{C N}
$$

But once we have dispersed values of $\tilde{C}_{N}$ and $\tilde{C}_{m}$, how do we generate a load profile that's consistent?

This is more than an esoteric question; for example, we need dispersed loads if we want to know the UQ on other integral properties like maximum bending moment 


\section{Bad Idea 1: Scaling}

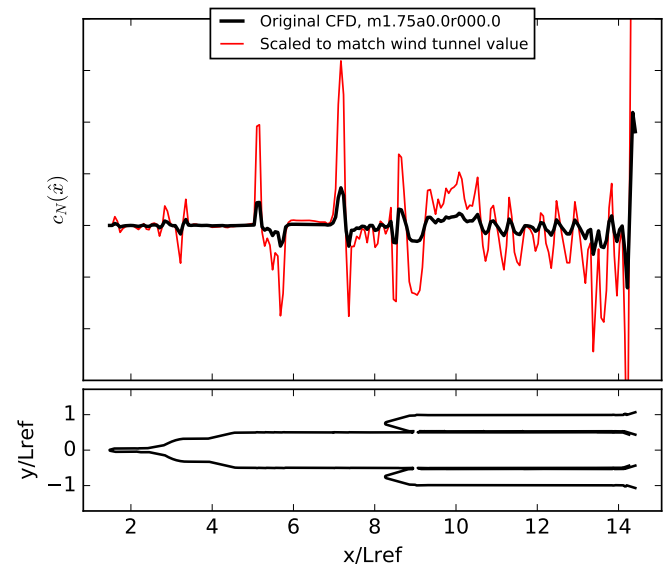

Directly scaled $C N$ for Mach 1.75, $\alpha=0^{\circ}, \beta=0^{\circ}$
- Scaling the entire profile has huge problems with small integrated loads

- Suppose the CFD value of the database is $C_{N}=0.001$ and the value measured in the wind tunnel is $\bar{C}_{N}=0.02$

- Scaling the value shifts the black load profile to the red one

- If CFD val. is $C_{N}=-0.001$, it gets much worse

- Also, doing this eliminates control over the pitching moment, $\bar{C}_{m}$ 


\section{Bad Idea 2: Constant Shift}

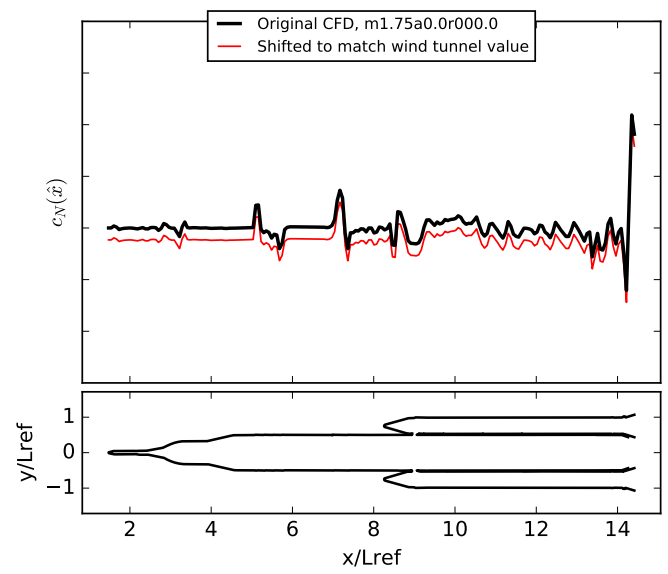

$C N$ with shift for Mach 1.75, $\alpha=0^{\circ}, \beta=0^{\circ}$
- Shifting the entire profile has similar problems

- Suppose the CFD value of the database is $C_{N}=0.001$ and the value measured in the wind tunnel is $\bar{C}_{N}=0.02$

- Shifting the value shifts the black load profile to the red one

- Loses track of all the places where the load should be zero

- For UQ, the resulting dispersion is (locally) too small

- If CFD val. is $C_{N}=-0.001$, no dramatic difference

- Also, doing this eliminates control over the pitching moment, $\bar{C}_{m}$ 


\section{Bad (but better) Idea 3: Linear Shift}

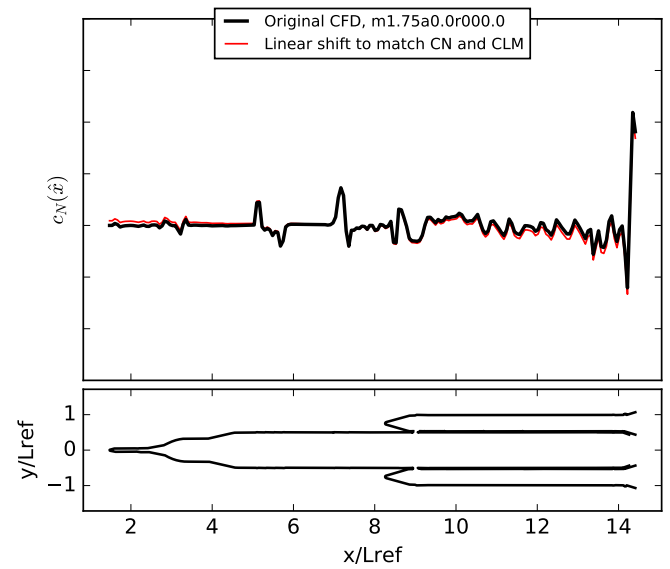

$C N$ at Mach $1.75, \alpha=0^{\circ}, \beta=0^{\circ}$, with linear shift
- Now matching $\bar{C}_{N}$ and $\bar{C}_{m}$

- Suppose the CFD value of the database is $C_{N}=0.001$ and the value measured in the wind tunnel is $\bar{C}_{N}=0.02$

- Wild shifts no longer apparent

- However, largest adjustments are always at the nose and tail

- All zero crossings are shifted

- Better than other two, but this doesn't utilize any specific information about the vehicle or conditions 


\section{Proper Orthogonal Decomposition}

Concept: Use a family of discretized line loads and use first few POD modes as candidate adjustment functions

$$
\mathbf{c}_{N, i}=\left[\begin{array}{c}
c_{N, i, 1} \\
c_{N, i, 2} \\
\vdots \\
c_{N, i, n}
\end{array}\right]=\left[\begin{array}{c}
c_{N}\left(\hat{x}_{1}, M_{i}, \alpha_{i}, \beta_{i}\right) \\
c_{N}\left(\hat{x}_{2}, M_{i}, \alpha_{i}, \beta_{i}\right) \\
\vdots \\
c_{N}\left(\hat{x}_{n}, M_{i}, \alpha_{i}, \beta_{i}\right)
\end{array}\right]
$$

Here $i$ represents the flight condition index

Now take several of these "snapshot" vectors and put them into a matrix

$$
\mathbf{C}_{N}=\left[\begin{array}{llll}
\mathbf{c}_{N, 1} & \mathbf{c}_{N, 2} & \cdots & \mathbf{c}_{N, n}
\end{array}\right]
$$

Then perform a singular value decomposition of the $n \times m$ matrix $\mathbf{C}_{N}$

$$
\mathbf{C}_{N}=\boldsymbol{\Phi}_{N} \boldsymbol{\Sigma}_{N} \mathbf{V}_{N}^{T}
$$

Dimensions: $\boldsymbol{\Phi}_{N} \in \mathbb{R}^{m \times m}, \boldsymbol{\Sigma}_{N} \in \mathbb{R}^{m \times n}, \mathbf{V}_{N} \in \mathbb{R}^{n \times n}$ 


\section{Adjustment Modes from POD}

$$
\mathbf{C}_{N}=\mathbf{\Phi}_{N} \boldsymbol{\Sigma}_{N} \mathbf{V}_{N}^{T}
$$

The columns of $\boldsymbol{\Phi}_{N}$ are basically line load profiles

$$
\hat{\phi}_{N, k}=\left[\begin{array}{c}
\phi_{N, k, 1} \\
\phi_{N, k, 2} \\
\vdots \\
\phi_{N, k, n}
\end{array}\right]
$$

with some special properties

$$
\left\|\hat{\phi}_{N, k}\right\|=1, \quad \hat{\phi}_{N, j} \cdot \hat{\phi}_{N, k}= \begin{cases}1 & j=k \\ 0 & j \neq k\end{cases}
$$

The matrix $\Sigma_{N}$ is a rectangular matrix with singular values along its diagonal

$$
\boldsymbol{\Sigma}_{N}=\left[\begin{array}{cccc}
\sigma_{N, 1} & 0 & \cdots & 0 \\
0 & \sigma_{N, 2} & & 0 \\
\vdots & & \ddots & \vdots \\
0 & 0 & \cdots & \sigma_{N, m} \\
0 & 0 & \cdots & 0 \\
\vdots & \vdots & & \vdots \\
0 & 0 & \cdots & 0
\end{array}\right]
$$

Singular values give relative energy content in each mode, $\sigma_{N, 1} \geq \ldots \sigma_{N, m} \geq 0$

Then we can select the first $K \leq m$ modes and use a linear combination to adjust the line load profile

$$
\bar{c}_{N, i}(\hat{x})=c_{N, i}(\hat{x})+\sum_{k=1}^{K} a_{k} \hat{\phi}_{N, k}(\hat{x})
$$




\section{POD Snapshots at Mach 1.30}
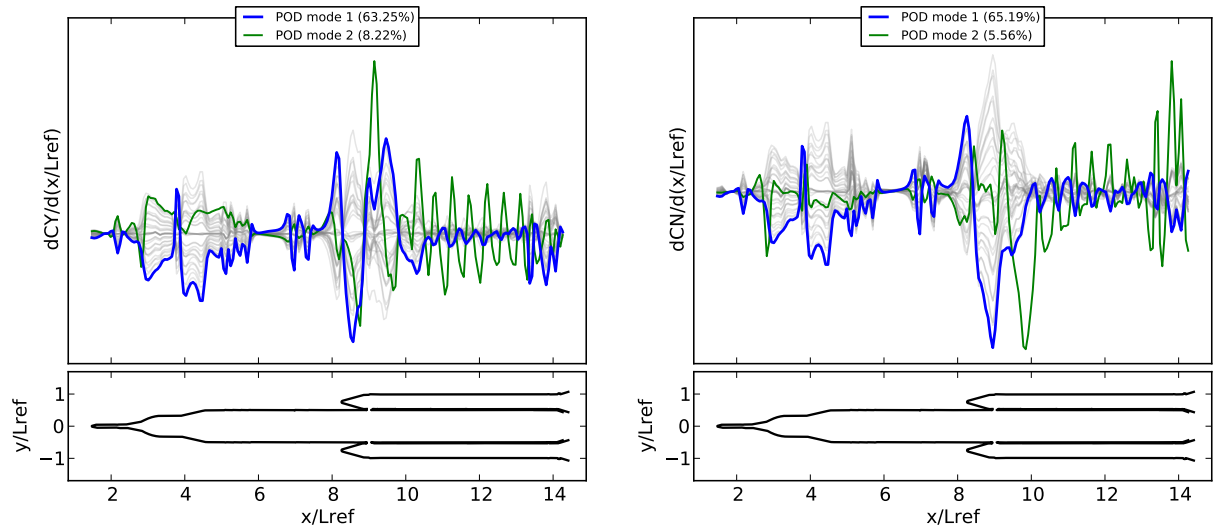

CY snapshots

$C N$ snapshots 


\section{Optimal Weights for POD Adjustment}

Select the first $K \leq m$ POD modes to adjust the line load profile for case $i$

$$
\bar{c}_{N, i}(\hat{x})=c_{N, i}(\hat{x})+\sum_{k=1}^{K} a_{k} \hat{\phi}_{N, k}(\hat{x})
$$

Now we have $K$ degrees of freedom (usually $K \approx 10$ works well) and only two constraints

$$
\begin{array}{ll}
\Delta C_{N, k}=\int_{\hat{x}_{1}}^{\hat{x}_{2}} \hat{\phi}_{N, k}(\hat{x}) \mathrm{d} \hat{x} & \bar{C}_{N}=C_{N}+\sum_{k=1}^{K} a_{k} \Delta C_{N, k} \\
\Delta C_{m, k}=\int_{\hat{x}_{1}}^{\hat{x}_{2}}\left(\hat{x}-\hat{x}_{M R P}\right) \hat{\phi}_{N, k}(\hat{x}) \mathrm{d} \hat{x} & \bar{C}_{m}=C_{m}+\sum_{k=1}^{K} a_{k} \Delta C_{m, k}
\end{array}
$$

Our solution is to minimize a weighted $L_{2}$ norm of the total adjustment

$$
\min _{\mathbf{a} \in \mathbb{R}^{K}} f(\mathbf{a})=\sum_{k=1}^{K} w_{k} a_{k}^{2}
$$

Using maximum absolute value (questionable) and singular value (pretty logical) to set the weights

$$
v_{k}=\max _{\hat{x} \in\left[\hat{x}_{1}, \hat{x}_{2}\right]}\left|\hat{\phi}_{N, k}(\hat{x})\right|=\left\|\hat{\phi}_{N, k}\right\|_{\infty} \quad \quad w_{k}=v_{k} / \sigma_{N, k}
$$




\section{Optimal Weights for POD Adjustment}

One reason for this setup is that it can be easily solved using lagrange multipliers

$$
\begin{aligned}
F\left(a_{1}, \ldots, a_{K}, \lambda_{1}, \lambda_{2}\right)=\lambda_{1}( & \left.\bar{C}_{N}-C_{N}-\sum_{k=1}^{K} a_{k} \Delta C_{N, k}\right)+ \\
& \lambda_{2}\left(\bar{C}_{m}-C_{m}-\sum_{k=1}^{K} a_{k} \Delta C_{m, k}\right)+\sum_{k=1}^{K} w_{k} a_{k}^{2}
\end{aligned}
$$

This leads to a linear system of equations with a predictable format

$$
\left[\begin{array}{cccccc}
\Delta C_{N, 1} & \Delta C_{N, 2} & \cdots & \Delta C_{N, K} & 0 & 0 \\
\Delta C_{m, 1} & \Delta C_{m, 2} & \cdots & \Delta C_{m, K} & 0 & 0 \\
-2 w_{1} & 0 & \cdots & 0 & \Delta C_{N, 1} & \Delta C_{m, 1} \\
0 & -2 w_{2} & \ddots & \vdots & \Delta C_{N, 2} & \Delta C_{m, 2} \\
\vdots & \ddots & \ddots & 0 & \vdots & \vdots \\
0 & \cdots & 0 & -2 w_{K} & \Delta C_{N, K} & \Delta C_{m, K}
\end{array}\right]\left[\begin{array}{c}
a_{1} \\
a_{2} \\
\vdots \\
a_{K} \\
\lambda_{1} \\
\lambda_{2}
\end{array}\right]=\left[\begin{array}{c}
\bar{C}_{N}-C_{N} \\
\bar{C}_{m}-C_{m} \\
0 \\
0 \\
\vdots \\
0
\end{array}\right]
$$

There is a similar system for $c_{Y}(\hat{x})$ and one with one less row and column for $c_{A}(\hat{x})$ 


\section{Sample Adjustments of $C_{A}$}
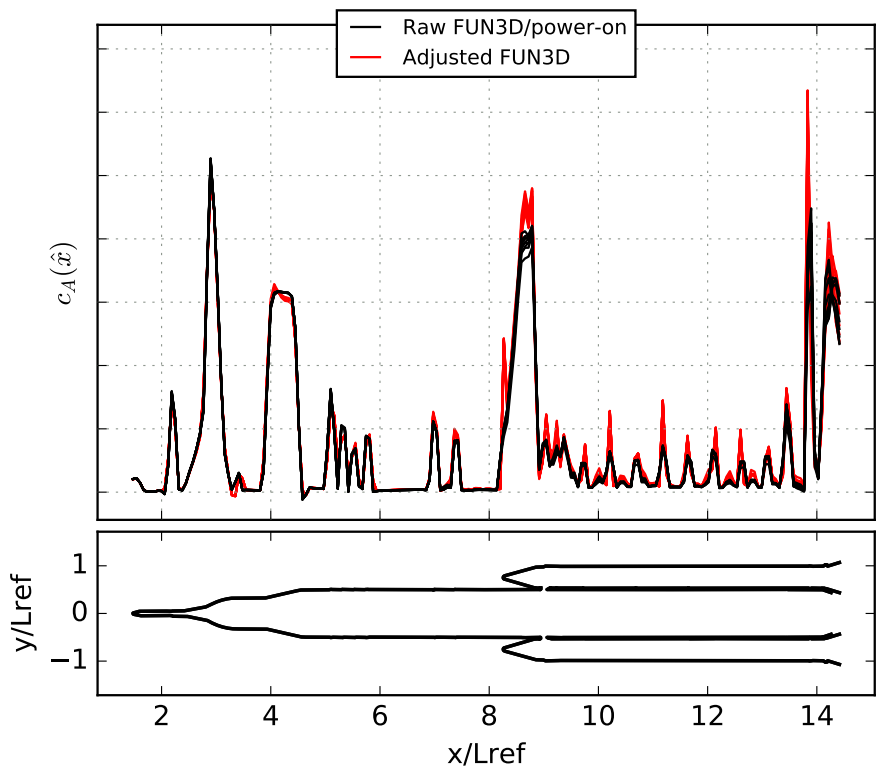

Mach 1.30, $\alpha_{t}=4^{\circ}, \beta \geq 0^{\circ}$ original (black) and adjusted (red) loads 


\section{Sample Adjustments of $C_{Y}$}

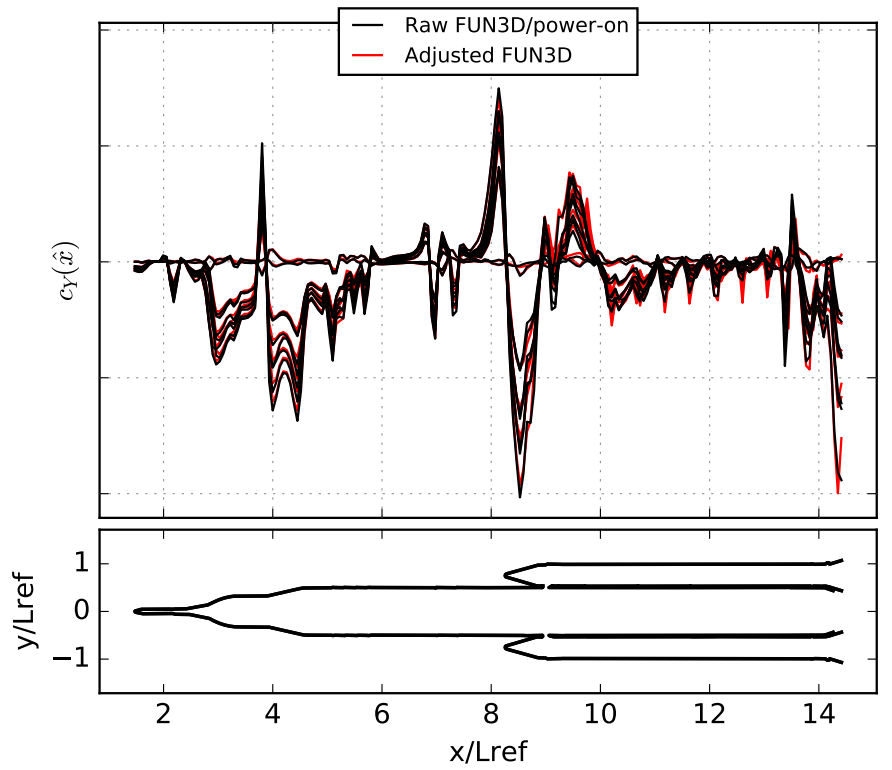

Mach 1.30, $\alpha_{t}=4^{\circ}, \beta \geq 0^{\circ}$ original (black) and adjusted (red) loads 


\section{Sample Adjustments of $C_{N}$}



Mach 1.30, $\alpha_{t}=4^{\circ}, \beta \geq 0^{\circ}$ original (black) and adjusted (red) loads 


\section{Uncertainty Quantification}

The idea is simple: use the UQ from the force \& moment database

$$
\begin{aligned}
& \tilde{C}_{N}=\bar{C}_{N}+\varepsilon_{C N} U_{C N} \\
& \tilde{C}_{m}=\bar{C}_{m}+\varepsilon_{C L M} U_{C L M}+\left(\hat{x}_{M R P}-\hat{x}_{C g}\right) \varepsilon_{C N} U_{C N}
\end{aligned}
$$

Here $\varepsilon_{C N}$ and $\varepsilon_{C L M}$ are randomly dispersed variables and $U_{C N}$ and $U_{C L M}$ are the quantifications of uncertainty in normal force and pitching moment, respectively

That's basically it; now just readjust the line loads to match $\tilde{C}_{N}$ and $\tilde{C}_{m}$.

To use properly, structures team or other customers should really do analysis for each trajectory in the Monte Carlo instead of just once for each flight condition 


\section{Dispersed $c_{N}(\hat{x})$ at Mach 1.75, $\alpha=4^{\circ}, \beta=0^{\circ}$}

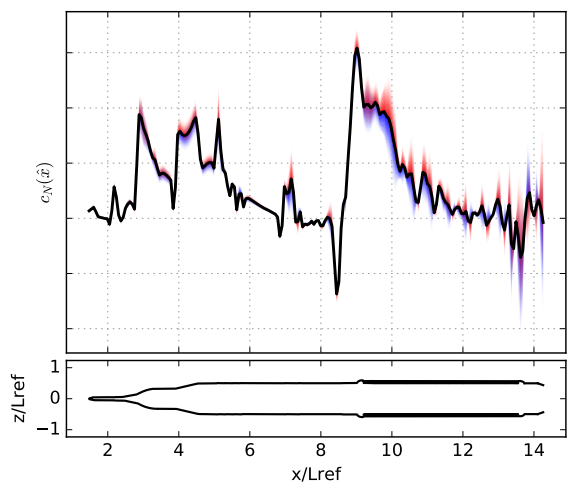

R: Increase in $C_{N}$, B: Decrease in $C_{N}$

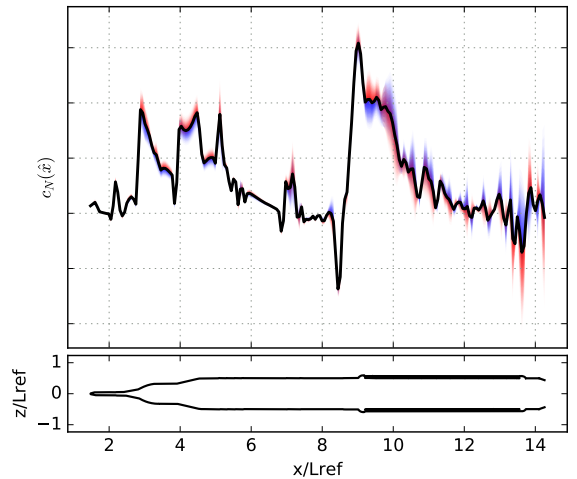

$\mathrm{R}$ : Increase in $C_{m}$, B: Decrease in $C_{m}$

- Each curve is the $c_{N}(\hat{x})$ profile for one combination of $C_{N}$ and $C_{m}$

- Enough curves here to to make it look like a PDF at each $x$ value

- The two charts show the same data but colored in two different ways

- Some regions correlate better to $C_{N}$ and some to $C_{m}$ 


\section{Dispersed $c_{Y}(\hat{x})$ at Mach 1.75, $\alpha=4^{\circ}, \beta=0^{\circ}$}



R: Increase in $C_{Y}$, B: Decrease in $C_{Y}$

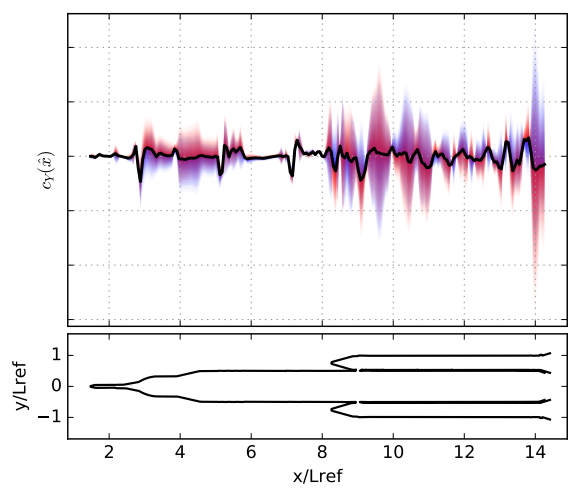

$\mathrm{R}$ : Increase in $C_{n}$, B: Decrease in $C_{n}$

- Each curve is the $c_{N}(\hat{x})$ profile for one combination of $C_{Y}$ and $C_{n}$

- Enough curves here to to make it look like a PDF at each $x$ value

- The two charts show the same data but colored in two different ways

- In this case, local loads correlate with $C_{Y}$ and not $C_{n}$ 


\section{Conclusions}

- Relatively simple, very reliable method to adjust CFD-based line loads to be consistent with wind tunnel integrated loads

- This paper suggests a method, but it has several opportunities to make other decisions

- No need for additional CFD solutions

- Easily extended to create an uncertainty quantification that is consistent with a force \& moment UQ

- Technique easily extended:

- Adjust/disperse surface pressures (and skin friction) instead of line loads to match all six F\&M at once

- Add more dispersion modes that do not affect integrated F\&M 


\section{Acknowledgments}

- SLS Program; this work is part of the SLS Aero Task Team

- Other members of the NASA ARC/TNA SLS CFD Team:

- Jeff Onufer

- Tom Pulliam

- and many previous members

- NASA Advanced Supercomputing facilities

- NASA Langley SLS CFD Team

- NASA Engineering \& Safety Center (NESC) for discussions and reviews 